\title{
Pharmacodynamic Parameters of Immunosuppressive Drugs Are Not Correlated with Age, Duration of Dialysis, Percentage of Lymphocytes or Lymphocyte Stimulation Index in Renal Transplant Recipients
}

\author{
Kentaro Sugiyama, ${ }^{*, a}$ Kazuya Isogai, ${ }^{a}$ Akira Toyama, ${ }^{a}$ Hiroshi Satoh, ${ }^{a}$ Kazuhide Saito, ${ }^{b}$ \\ Yuki Nakagawa, ${ }^{b}$ Masayuki TAsaki, ${ }^{b}$ Kota TAKAhashi, ${ }^{b}$ Noriko SAIto, ${ }^{c}$ and Toshihiko Hirano ${ }^{d}$ \\ ${ }^{a}$ Division of Pharmacy, Niigata University Medical and Dental Hospital; 754 Asahimachi-dori 1-bancho, Chuo-ku, \\ Niigata 951-8520, Japan: ${ }^{b}$ Division of Urology, Graduate School of Medical and Dental Sciences, Niigata University; \\ ${ }^{c}$ Division of Clinical Nephrology, Rheumatology, Respiratory Medicine, and Infection Control and Prevention, Graduate \\ School of Medical and Dental Sciences, Niigata University; 757 Asahimachi-dori 1-bancho, Chuo-ku, Niigata 951-8510, \\ Japan: and ${ }^{d}$ Department of Clinical Pharmacology, School of Pharmacy, Tokyo University of Pharmacy and Life Science; \\ 1432-1 Horinouchi, Hachioji 192-0392, Japan.
}

Received July 28, 2008; accepted September 5, 2008; published online September 8, 2008

The lymphocyte immunosuppressant-sensitivity test (LIST) has been reported extensively as being able to estimate the pharmacological efficacy of immunosuppressive drugs in individual patients. This study measured the $\mathrm{IC}_{50}$ values for 6 drugs, cyclosporine, tacrolimus, methylprednisolone, 6-mercaptopurine, mycophenolic acid, and mizoribine, against mitogen-induced proliferation of peripheral-blood lymphocytes in 29 renal transplant recipients. We also examined relationship between the $\mathrm{IC}_{50}$ values and 4 factors; age, duration of dialysis, percentage of lymphocytes in total white blood cells, and blastogenesis stimulation index by mitogen. There were no significant correlations between the $\mathrm{IC}_{50}$ values and these factors, except that the tacrolimus $\mathrm{IC}_{50}$ value was correlated weakly with the stimulation index $(p<0.05, r=-0.429)$. It was concluded that the pharmacological efficacy of immunosuppressive drugs cannot be inferred from the patient characteristics or their laboratory data. LIST is an effective method to elucidate the pharmacodynamic properties of immunosuppressive agents in individual renal transplant recipients without being influenced by the recipient clinical data.

Key words immunosuppressive therapy; renal transplantation; aging; lymphocyte immunosuppressant-sensitivity test

Immunosuppressive agents, including calcineurin inhibitors, exhibit individual variation in their therapeutic efficacies in allograft recipients. In general, therapeutic drug monitoring (TDM) of cyclosporine or tacrolimus is performed to examine the dosing of drugs that should be used to optimize immunosuppressive therapy. However, it is not always possible to prevent allograft rejection after transplantation regardless of TDM-based immunosuppressive therapy. This fact suggested that the large individual variation in clinical efficacy of immunosuppressants is attributed to individual differences in not only pharmacokinetic but also pharmacodynamic parameters of the drugs.

This study employed a lymphocyte immunosuppressant sensitivity test (LIST) to evaluate the pharmacodynamic properties of immunosuppressive agents. ${ }^{1-7)}$ LIST is an assay to evaluate the pharmacological efficacy of immunosuppressive drugs in-vitro using the peripheral-blood mononuclear cells (PBMCs) of transplant recipients. In this assay, PBMCs were incubated in the presence of concanavalin A and the immunosuppressive agents, and the concentration-dependent inhibitory effects of the drugs on concanavalin A-stimulated PBMC blastogenesis were measured. It was reported previously that the $\mathrm{IC}_{50}$ values of prednisolone against blastogenesis varied widely among patients, and the $\mathrm{IC}_{50}$ values correlated with the clinical efficacy of this drug in renal transplantation. ${ }^{1)}$ On the other hand, the pharmacological efficacy of immunosuppressive agents may also be affected by the immunological state of patients. Agerelated deterioration in immune function, termed immunosenescence, has been recognized for decades. Hemodialysis or peritoneal dialysis has been suggested to contribute to oxida- tive stress in patients, and dialysis may modulate immune functions through an increase in reactive oxygen species. The percentage of lymphocytes to total white blood cells and blastogenesis stimulation index (i.e., the ratio of lymphocyte proliferation stimulated and unstimulated by concanavalin A) are also related to immunoactivity. If the efficacy (pharmacodynamics) of immunosuppressive agents correlates with age, duration of dialysis, percentage of lymphocytes, or stimulation index, the correlations will contribute to the development of tailored immunosuppressive therapies.

In order to examine the relationship between the efficacy of immunosuppressive drugs and patient characteristics or laboratory diagnostic data, in the present study the $\mathrm{IC}_{50}$ values of 6 drugs, cyclosporine, tacrolimus, methylprednisolone, 6-mercaptopurine, mycophenolic acid, and mizoribine were measured in 29 renal transplant recipients using LIST. Then, the data of $\mathrm{IC}_{50}$ values were examined for their relation with each 4 factors; age, duration of dialysis, percentage of lymphocytes in total white blood cells, and blastogenesis stimulation index by mitogen.

\section{MATERIALS AND METHODS}

Materials Cyclosporine, tacrolimus, and mizoribine were kindly provided by Novartis (Basel, Switzerland), Astellas (Tokyo, Japan), and Asahi Kasei Pharma (Tokyo, Japan), respectively. Methylprednisolone and 3-(4,5-dimethylthiazol-2-yl)-2,5-diphenyl tetrazolium bromide (MTT) were obtained from Sigma Aldrich (St. Louis, MO, U.S.A.). Mycophenolic acid and 6-mercaptopurine were purchased from Wako Chemical (Osaka, Japan). Ficoll-Paque was ob- 
tained from Amersham Pharmacia Biotech (Buckinghamshire, U.K.). RPMI-1640 medium, fetal bovine serum, and Hank's balanced salt solution were obtained from Gibco Laboratories (Rockville, MD, U.S.A.). All other reagents were of the highest grade available.

Subjects Twenty nine renal transplant recipients (21 males and 8 females), who were admitted to Niigata University Medical and Dental Hospital, were enrolled in the present study. After written informed consent was obtained, heparinized venous blood $(20 \mathrm{ml})$ was taken from the recipients just before renal transplantation. The mean (S.D.) age of the recipients was 34.9 (13.0) years, while the median was 31 years (range 14 to 65 years). The mean (S.D.) value for the duration of dialysis prior to renal transplant was 4.4 (5.4) years, and the median was 2.5 years (ranged from 0 to 20.0 years) (Table 1).

All of the transplant recipients received renal allografts from living donors after blood-sampling for analysis of their PBMC-response to immunosuppressive agents in vitro. Renal transplantation in these patients was performed from July 2002 to October 2004 at Niigata University Medical and Dental Hospital.

Evaluation of Immunosuppressant Pharmacological Efficacy by LIST with MTT Assays The procedure of LIST using MTT assays was described previously. ${ }^{2-5)}$ In brief, PBMCs isolated from venous blood of the patients were suspended in RPMI-1640 medium containing 10\% fetal bovine serum to a cell density of $1 \times 10^{6}$ cells $/ \mathrm{ml}$. Concanavalin A was added at a final mitogen concentration of $5.0 \mu \mathrm{g} / \mathrm{ml}$. Subsequently, immunosuppressive drugs were added individually at a range of concentrations. After $96 \mathrm{~h}$ incubation in an atmosphere $5 \% \mathrm{CO}_{2}$ at $37^{\circ} \mathrm{C}, 10 \mu \mathrm{l}$ of $5 \mathrm{mg} / \mathrm{ml}$ MTT solution was added and then the cultures were incubated for a further $4-5 \mathrm{~h}$. The cultures were centrifuged at $375 \times \boldsymbol{g}$ for $5 \mathrm{~min}$ to obtain precipitated formazan, and aliquots of the supernatant were removed. Dimethylsulfoxide was added and followed by shaking for $10 \mathrm{~min}$ to dissolve the formazan crystals and the absorbance of the solution was measured at $550 \mathrm{~nm}$. The percentage of blastogenesis was plotted against the drug concentrations and the $\mathrm{IC}_{50}$ values were determined from the concentration-response curves. The percentage of lymphocytes in the peripheral leukocytes was measured at the same time. The index was calculated from the formula: absorbance at $550 \mathrm{~nm}$ in well in presence of stimulant/absorbance at $550 \mathrm{~nm}$ in well in the absence of stimulant. We also examined relationship between the $\mathrm{IC}_{50}$ values and 4 factors; age, duration of dialysis, percentage of lymphocytes in total white blood cells, and blastogenesis stimulation index by mitogen.

The study was approved by the Ethical Review Board of the Medical Faculty of Niigata University.

Statistical Analysis Because of their skewed distribution, the raw $\mathrm{IC}_{50}$ data were log-transformed prior to statistical analysis. The correlation coefficient between each of immunosuppressant $\mathrm{IC}_{50}$ values and the age of transplant recipients was analyzed using Pearson's test. Statistical analysis software SPSS 11.0J was used for the analysis (SPSS Japan Inc.).

\section{RESULTS}

The pharmacological efficacy of each of immunosuppressive agents on mitogen-induced blastogenesis in PBMCs was evaluated in-vitro in 29 renal transplant recipients by LIST with MTT assays. Typical concentration-response curves for cyclosporine and tacrolimus against the blastogenesis of PBMCs obtained from one renal transplant recipient are shown in Fig. 1. The mean (S.D.) of cyclosporine $\mathrm{IC}_{50}$ values

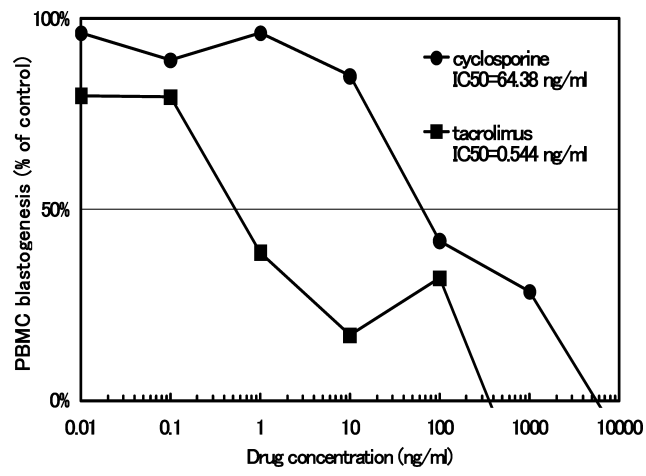

Fig. 1. Typical Concentration-Response Curves for Calcineurin Inhibitors on Concanavalin A-Stimulated Blastogenesis of PBMCs from One Transplant Recipient Estimated by LIST Followed by MTT Assays

Table 1. $\mathrm{IC}_{50}$ Values for Each Immunosuppressive Agent and Clinical Characteristics of Renal Transplant Recipients $(n=29)$

\begin{tabular}{|c|c|c|c|c|}
\hline \multirow{2}{*}{ Immunosuppressive agents } & \multicolumn{4}{|c|}{$\mathrm{IC}_{50}$ values $(\mathrm{ng} / \mathrm{ml})$} \\
\hline & Mean \pm S.D. & Median & Minimum & Maximum \\
\hline Cyclosporine & $1012.6 \pm 3084.1$ & 158.5 & 0.07 & 16377.8 \\
\hline Tacrolimus & $20.5 \pm 82.5$ & 0.35 & 0.000168 & 442.0 \\
\hline Methylprednisolone & $2286.2 \pm 5214.1$ & 715.5 & 2.15 & 26685.0 \\
\hline 6-Mercaptopurine & $800.9 \pm 3152.0$ & 50.7 & 0.0017 & 17019.0 \\
\hline Mycophenolic acid & $79.6 \pm 30.5$ & 73.3 & 21.8 & 161.0 \\
\hline Mizoribine & $20265.8 \pm 17621.8$ & 14528.0 & 867.7 & 66089.2 \\
\hline Characteristics & Mean \pm S.D. & Median & Minimum & Maximum \\
\hline Age of renal transplant recipients (years) & $34.9 \pm 13.0$ & 31 & 14 & 65 \\
\hline Duration of dialysis (years) & $4.4 \pm 5.4$ & 2.5 & 0 & 20 \\
\hline Percentage of lymphocytes (\%) & $29.3 \pm 6.9$ & 28.3 & 16.1 & 44.6 \\
\hline Stimulation index & $2.46 \pm 0.54$ & 2.33 & 1.56 & 3.73 \\
\hline
\end{tabular}


Table 2. Relationship between $\mathrm{IC}_{50}$ Values for Each Immunosuppressive Agent and Patient-Related Factors as Analyzed by Pearson's Coefficient Correlation Test

\begin{tabular}{lcccc}
\hline \hline & \multicolumn{4}{c}{ Patient-related factors } \\
\cline { 2 - 5 } $\begin{array}{c}\text { Immunosuppressive } \\
\text { agents }\end{array}$ & $\begin{array}{c}\text { Age of renal } \\
\text { transplant } \\
\text { recipients } \\
\text { (years) }\end{array}$ & $\begin{array}{c}\text { Duration } \\
\text { of dialysis } \\
\text { (years) }\end{array}$ & $\begin{array}{c}\text { Percentage of } \\
\text { lymphocytes } \\
(\%)\end{array}$ & $\begin{array}{c}\text { Stimulation } \\
\text { index }\end{array}$ \\
\hline Cyclosporine & 0.018 & -0.026 & 0.042 & -0.112 \\
Tacrolimus & 0.027 & 0.037 & -0.041 & $-0.429 *$ \\
Methylprednisolone & 0.18 & -0.19 & -0.296 & -0.313 \\
6-Mercaptopurine & -0.097 & 0.081 & 0.032 & -0.217 \\
Mycophenolic acid & -0.071 & 0.265 & 0.015 & -0.246 \\
Mizoribine & -0.19 & 0.13 & 0.146 & -0.119 \\
\hline \multicolumn{1}{c}{$* 0.05}$. & & & & \\
\hline
\end{tabular}

in 29 recipients before transplantation was 1012.6 (3084.1) $\mathrm{ng} / \mathrm{ml}$, and the median was $158.5 \mathrm{ng} / \mathrm{ml}$. The $\mathrm{IC}_{50}$ values ranged from 0.07 to $16377.8 \mathrm{ng} / \mathrm{ml}$. The mean (S.D.) of tacrolimus $\mathrm{IC}_{50}$ values was $20.5(82.5) \mathrm{ng} / \mathrm{ml}$, and the median was $0.35 \mathrm{ng} / \mathrm{ml}$. The $\mathrm{IC}_{50}$ values ranged from 0.000168 to $442.0 \mathrm{ng} / \mathrm{ml}$ (Table 1).

The mean (S.D.) of methylprednisolone $\mathrm{IC}_{50}$ values in the 29 recipients was $2286.2(5214.1) \mathrm{ng} / \mathrm{ml}$, and the median was $715.5 \mathrm{ng} / \mathrm{ml}$. The $\mathrm{IC}_{50}$ values ranged from 2.15 to 26685.0 $\mathrm{ng} / \mathrm{ml}$. The mean (S.D.) of 6-mercaptopurine $\mathrm{IC}_{50}$ values in the 29 recipients was $800.9(3152.0) \mathrm{ng} / \mathrm{ml}$, and the median was $50.7 \mathrm{ng} / \mathrm{ml}$. The $\mathrm{IC}_{50}$ values ranged from 0.0017 to $17019.0 \mathrm{ng} / \mathrm{ml}$. The mean (S.D.) of mycophenolic acid $\mathrm{IC}_{50}$ values was $79.6(30.5) \mathrm{ng} / \mathrm{ml}$, and the median was 73.3 $\mathrm{ng} / \mathrm{ml}$. The $\mathrm{IC}_{50}$ values ranged from 21.8 to $161.0 \mathrm{ng} / \mathrm{ml}$. The mean (S.D.) of mizoribine $\mathrm{IC}_{50}$ values was 20265.8 $(17621.8) \mathrm{ng} / \mathrm{ml}$, and the median was $14528.0 \mathrm{ng} / \mathrm{ml}$. The $\mathrm{IC}_{50}$ values ranged from 867.7 to $66089.2 \mathrm{ng} / \mathrm{ml}$ (Table 1). Thus, the individual differences in the pharmacological efficacy of mycophenolic acid were smaller compared with that of other purine-synthesis inhibitors in renal transplant recipients.

The mean (S.D.) of the percentage of lymphocytes in PBMCs was $29.3 \%$ (6.9), and the median was $28.3 \%$ (range 16.1 to $44.6 \%$ ). The mean (S.D.) of stimulation indices in concanavalin A stimulated PBMCs was 2.46 (0.54), and the median of which was 2.33 (ranged from 1.56 to 3.73) (Table $1)$.

There was no statistically significant correlation between the log-transformed $\mathrm{IC}_{50}$ values of each immunosuppressive agent and the age of renal transplant recipients. The logtransformed $\mathrm{IC}_{50}$ values for each immunosuppressive agent and the durations of dialysis before transplantation were not statistically correlated. It was also reported previously that there was no significant correlation between the $\mathrm{IC}_{50}$ values of glucocorticoids, as assessed by the ${ }^{3} \mathrm{H}$-thymidine assay procedures, and the duration of dialysis in renal transplant recipients. ${ }^{1)}$ Similarly, the log-transformed $\mathrm{IC}_{50}$ values for each immunosuppressive agent were not correlated with the percentages of peripheral-blood lymphocytes (Table 2).

Furtheremore, there were significant correlation between age and percentage of lymphocytes $(p<0.05, r=-0.475)$, age and stimulation index $(p<0.05, r=-0.354)$, or dulation of dialysis and stimulation index $(p<0.05, r=-0.292)$.
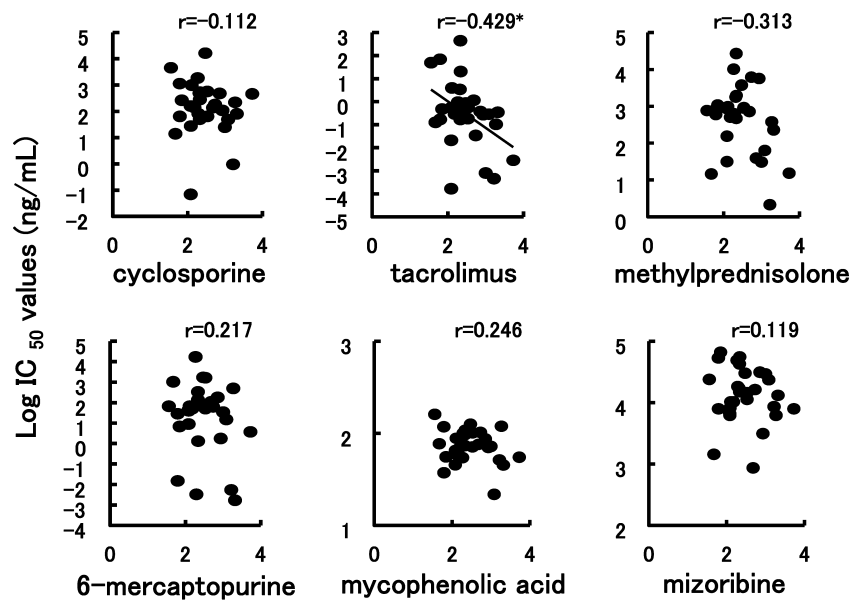

Fig. 2. Relationship between the $\mathrm{IC}_{50}$ Values for Each Immunosuppressive Agent and the Stimulation Indices in Concanavalin A-Stimulated PBMCs of Renal Transplant Recipients before Operation

Finally, the relationship between $\mathrm{IC}_{50}$ values for immunosuppressive drugs and stimulation indices were examined. These values, except for the $\mathrm{IC}_{50}$ value for tacrolimus, were not significantly correlated (Table 2 ). There was only a weak correlation between the tacrolimus $\mathrm{IC}_{50}$ value and the stimulation indices $(p<0.05, r=-0.429)$. The relationships between the $\mathrm{IC}_{50}$ values of each immunosuppressive agent and the stimulation indices are shown in Fig. 2.

\section{DISCUSSION}

The data from the present study suggested the $\mathrm{IC}_{50}$ values of immunosuppressive agents are not influenced by the age, duration of dialysis, percentage of peripheral-blood lymphocytes, or stimulation index in renal transplant recipients. Many studies, including the preceding study by the present authors, ${ }^{7)}$ have shown that lymphocyte sensitivity to immunosuppressive drugs in-vitro is correlated with the clinical efficacy of the drug in renal transplantation and immunological disorders. ${ }^{89}$ ) The previous study showed that the $\mathrm{IC}_{50}$ values of tacrolimus and cyclosporine varied widely in renal transplant recipients. $\left.{ }^{6}\right)$ Furthermore, the $\mathrm{IC}_{50}$ value of cyclosporine assessed by LIST with MTT assays was significantly lower than the cyclosporine pharmacological efficacy assessed by LIST with ${ }^{3} \mathrm{H}$-thymidine assays. $\left.{ }^{2}\right)$ The present data on the pharmacological efficacy of cyclosporine and tacrolimus was almost the same as those of the previous reports. ${ }^{2,6)}$

We found that there was only a weak correlation between the tacrolimus $\mathrm{IC}_{50}$ values and these stimulation indices, while the stimulation indices were not significantly correlated with the $\mathrm{IC}_{50}$ values of other drugs (Fig. 2, Table 2). It has been known that tacrolimus specifically inhibit $\mathrm{T}$ cell activation via impairment of calcineurin activity, ${ }^{10)}$ and therefore, the drug might exhibit relatively a low efficacy to suppress proliferation of PBMCs with low $\mathrm{T}$ cell activity. Thus, the efficacy of tacrolimus to inhibit proliferation might be high in PBMCs with high T cell activities (high stimulation indices) and vice versa. These backgrounds of tacrolimus action targeted to $\mathrm{T}$ cells may be related, at least partially, to a 
negative correlation between the tacrolimus $\mathrm{IC}_{50}$ values and the stimulation indices we presently observed. Cyclosporine is also known to block $\mathrm{T}$ cell activation by inhibiting calcineurin activity via binding to the other binding protein cyclophilin, but the immunosuppressive effect of cyclosporine is known to be somewhat different from that of tacrolimus. ${ }^{10}$ )

In this study, individual deviation of the mycophenolic acid pharmacological efficacies assigned by LIST was smallest comparing with the other immunosupprressive agents. In our previous report, we have measured $\mathrm{IC}_{50}$ values of mycophenolic acid, azathioprine and mizoribine by LIST with MTT assay procedure in renal transplant recipients and healthy subjects, and the results obtained were consistent with those of the present study. ${ }^{3)}$ There are two major pathways of purine synthesis, and cell types and tissues can be classified according to their dependence on the de-novo and salvage pathways of purine synthesis. Lymphocytes show extreme dependence on the de-novo synthesis. Mycofenolate mofetil is a potent, non-competitive, reversible inhibitor of the enzyme inosine mono-phosphate dehydrogenase (IMPDH), and therefore, this drug selectively suppresses the proliferation of both $\mathrm{T}$ and B lymphocytes. ${ }^{11)}$ Thus, the individual variation of the anti-lymphoproliferative efficacy of IMPDH-inhibitors among patients should be minimal. On the other hand, Azathioprine and its active metabolite, 6-mercaptopurine, are reported to block several enzymes involved in purine-synthesis through comparative inhibition, ${ }^{12}$ which may result in large individual variation of the anti-PBMC effect of Azathioprine, as we observed in previous study. Mizoribine is also known to inhibit IMPDH, but its efficacy was lower than that of mycophenolic acid, ${ }^{3)}$ which may result in relatively a wide individual variation of its efficacy.

It has been considered, in general, that immune responses decline with aging. In young subjects, lymphocyte-proliferation abilities in response to phytohemagglutinin (PHA) stimulation were significantly higher than those in older subjects. ${ }^{13)}$ The total number of peripheral CD4 and CD8 lymphocytes in older subjects were also reported to be reduced significantly when compared with those of younger subjects. ${ }^{14)}$ Lymphocytes stimulated with PHA-L or PMA/ionomycin in older subjects tended to display reduced levels of IL-2 expressing cells compared with those in younger subjects. ${ }^{14)}$ Furthermore, lymphocytes stimulated with PHA-L expressed significantly higher number of IFN- $\gamma$ positive cells in CD4 and CD8 subsets compared with those in younger subjects. ${ }^{14)}$

We also found here that there were significant correlation between age and percentage of lymphocytes, age and stimulation indices, or dulation of dialysis and stimulation indices.

Thus, lymphocyte responses to several stimuli appear to relate to aging. However, in the present study, the pharmacological efficacies of immunosuppressive agents evaluated by the LIST procedure were not significantly correlated with the age of renal transplant recipients. Therefore, standardized dosing or selection of immunosuppressive therapy based on patient age might not be required for renal transplant recipients. There was a weak correlation between the pharmacological efficacy of tacrolimus and the stimulation indices in this assay $(p<0.05, r=-0.475)$; however, such a correlation was not observed in the efficacies of other drugs.

In summary, the present report showed that PBMC sensitivities to immunosuppressive drugs evaluated by the LIST procedure were not influenced by the basic characteristics of the recipients including age, duration of hemodialysis, and percentage of lymphocytes in PBMCs. Furthermore, most of the pharmacological efficacies of immunosuppressive drugs in-vitro were not correlated with the stimulation indices. Thus, the data supported the validity of LIST as a tool to estimate individual sensitivity to immunosuppressive drugs for prescribing individualized therapy in renal transplant recipients.

Acknowledgements This study was supported by grant from The Kidney Foundation, Japan (JKF 06-3).

\section{REFERENCES}

1) Hirano T., Oka K., Takeuchi H., Sakurai E., Matsuno N., Tamaki T., Kozaki M., Transplantation, 57, 1341-1348 (1994).

2) Sugiyama K., Arakawa K., Satoh H., Saito K., Takahashi K., Saito N., Hirano T., J. Immunoassay Immunochem., 27, 195-205 (2006).

3) Sugiyama K., Satoh H., Saito K., Takahashi K., Saito N., Hirano T., Transpl. Int., 18, 590-595 (2005).

4) Sugiyama K., Satoh H., Hirano T., J. Pharm. Pharmacol., 55, 393 398 (2003).

5) Sugiyama K., Kawada T., Satoh H., Hirano T., J. Pharm. Pharmacol., 53, 727-733 (2001).

6) Takeuchi H., Hirano T., Oka K., Mizumoto K., Akashi T., Sakurai E., Degawa T., Uchiyama M., Kozaki K., Matsuno N., Nagao T., Kozaki M., Transplant. Proc., 30, 36-39 (1998)

7) Hirano T., Int. Immunopharmacol., 7, 3-22 (2006).

8) Hearing S. D., Norman M., Probert C. S., Haslam N., Dayan C. M., Gut, 45, 382-388 (1999).

9) Dudley J., Truman C., McGraw M., Tizard J., Haque G., Bradley B., Nephrol. Dial. Transplant., 18, 403-410 (2003).

10) Plosker G. L., Foster R. H., Drugs, 59, 323-389 (2000).

11) Anthony C. A., "The Molecular Biology of Immunosuppression," ed. by Thompson A. W., John Wiley \& Sons, Ltd., N.Y., 1992, pp. 181209.

12) Joel G. H., Alfred G. G., Lee E. L., "Goodman \& Gilman's The Pharmacological Basis of Therapeutics," 9th ed., 1995.

13) Di Pietro R., Miscia S., Cataldi A., Rana R., Br. J. Haematol., 111, 1209-1214 (2000).

14) Schindowski K., Frohlich L., Maurer K., Muller W. E., Eckert A., Mech. Ageing Dev., 123, 375-390 (2002). 